\title{
On the $L_{\mathrm{x}}-L_{6 \mu \mathrm{m}}$ ratio as a diagnostic for Compton-thick AGN
}

\author{
I. Georgantopoulos ${ }^{1,2}$, E. Rovilos ${ }^{1}$, A. Akylas ${ }^{2}$, A. Comastri ${ }^{1}$, P. Ranalli ${ }^{1}$, \\ C. Vignali ${ }^{1}$, I. Balestra $^{3}$, R. Gilli ${ }^{1}$, and N. Cappelluti ${ }^{1}$ \\ 1 INAF-Osservatorio Astronomico di Bologna, via Ranzani 1, 40127 Bologna, Italy \\ e-mail: ioannis.georgantopoulos@oabo.inaf.it \\ 2 Institute of Astronomy \& Astrophysics, National Observatory of Athens, Palaia Penteli, 15236 Athens, Greece \\ 3 Max-Planck Institut für Extraterrestrische Physik, Giessenbachstrasse 2, 85748 Garching, Germany
}

Received 2 June 2011 / Accepted 19 July 2011

\section{ABSTRACT}

\begin{abstract}
As the mid-IR luminosity represents a good isotropic proxy of the AGN power, a low X-ray to mid-IR luminosity ratio is often claimed to be a reliable indicator of Compton-thick AGN. We assess the efficiency of the X-ray to mid-IR luminosity ratio diagnostic by examining the $12 \mu \mathrm{m}$ IRAS AGN sample (intrinsic $L_{2-10 \mathrm{keV}}>10^{42} \mathrm{erg} \mathrm{s}^{-1}$ ) for which high signal-to-noise ratio XMM-Newton observations are now available. We find that the vast majority (ten out of eleven) of the AGN that were classified as Compton-thick on the basis of X-ray spectroscopy by Brightman \& Nandra have a low $L_{\mathrm{X}} / L_{6 \mu \mathrm{m}}$ luminosity ratio, i.e. lower than a few percent of the average AGN ratio, as is typical of reflection-dominated Compton-thick sources. At low $L_{X} / L_{6 \mu \mathrm{m}}$ ratios, we also find a comparable number of AGN, most of which are heavily absorbed but not Compton-thick. This implies that although most Compton-thick AGN have low $L_{X} / L_{6 \mu \mathrm{m}}$ ratios, at least in the local, Universe, the converse is not necessarily true. We then extend our analysis to higher redshifts. We perform the same analysis in the Chandra Deep Field South, for which excellent quality Chandra (4 Ms) and XMM-Newton (3 Ms) X-ray spectra are available. We derive accurate X-ray luminosities for Chandra sources using X-ray spectral

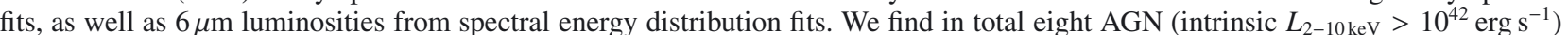
with low $L_{\mathrm{X}} / L_{6 \mu \mathrm{m}}$ ratios, after excluding one source where the $6 \mu \mathrm{m}$ emission primarily comes from star-formation. One of these sources has been already found to host a Compton-thick nucleus, while for another one at a redshift of $z=1.22$ we argue it is most likely Compton-thick on the basis of its combined Chandra and XMM-Newton spectrum. In agreement with the low redshift sample, we find a large number of non Compton-thick "contaminants" with low X-ray to mid-IR luminosity ratios. Our results suggest that a low $L_{X} / L_{6 \mu \mathrm{m}}$ ratio alone cannot help us to ascertain whether a Compton-thick AGN is present, albeit the majority of low $L_{\mathrm{X}} / L_{6 \mu \mathrm{m}}$ AGN are heavily obscured. More interestingly, the two most reliable Compton-thick AGN in the high redshift Universe have high $L_{\mathrm{X}} / L_{6 \mu \mathrm{m}}$ ratios, showing that this method cannot provide complete Compton-thick AGN samples.
\end{abstract}

Key words. X-rays: general - X-rays: diffuse background - X-rays: galaxies - infrared: galaxies

\section{Introduction}

The deep X-ray Universe has been probed to unparalleled depth thanks to the Chandra mission. The deepest Chandra X-ray surveys have measured an AGN sky density exceeding $10.000 \mathrm{deg}^{-2}$ (Alexander et al. 2003; Luo et al. 2008; Xue et al. 2011), probing a flux limit of $f_{2-10 \mathrm{keV}} \approx 1 \times 10^{-16} \mathrm{erg} \mathrm{cm}^{-2} \mathrm{~s}^{-1}$ (for a review see Brandt \& Hasinger 2005). In contrast, the optical surveys for QSOs reach sky densities of about a few hundred per square degree at a magnitude limit of $B=22 \mathrm{mag}$ (e.g. Wolf et al. 2003). Optical spectroscopic methods, which detect AGN using the [OIII] $(\lambda 5007 \AA)$ or the $[\mathrm{NeV}]$ lines ( $\lambda 3427 \AA$ ), (Hao et al. 2005; Bongiorno et al. 2010; Gilli et al. 2010), manage to recover a large fraction of low-luminosity AGN (Georgantopoulos \& Akylas 2010) but these can be applied across a limited redshift range, because these lines are redshifted outside the optical band at high redshifts. Therefore, hard $(2-10 \mathrm{keV}) \mathrm{X}$-ray surveys have been the most powerful means of detecting AGN and thus have provided the most detailed picture of the accretion history of the Universe (e.g. Ueda et al. 2003; La Franca et al. 2005; Aird et al. 2010).

However, even the efficient hard X-ray surveys may be missing a substantial fraction of the most heavily obscured sources, the Compton-thick AGN, which have column densities
$>10^{24} \mathrm{~cm}^{-2}$. At these high column densities, the attenuation of $\mathrm{X}$-rays is mainly due to Compton scattering rather than photoelectric absorption. Although only a few dozen of Comptonthick AGN have been identified in the local Universe (see Comastri 2004, for a review), there is concrete evidence that their number is much higher. The peak of the X-ray background at $20-30 \mathrm{keV}$ (e.g. Frontera et al. 2007; Churazov et al. 2007; Moretti et al. 2009) can be reproduced by invoking a significant number of Compton-thick sources at moderate redshifts. However, the exact density of Compton-thick sources required by X-ray background synthesis models still remains unclear (Gilli et al. 2007; Sazonov et al. 2008; Treister et al. 2009a; Ballantyne et al. 2011). Additional evidence of a numerous Compton-thick population comes from the directly measured space density of black holes in the local Universe (see Soltan 1982). It is found that this space density is a factor of 1.5-2 higher than predicted by the X-ray luminosity function (Marconi et al. 2004; Merloni \& Heinz 2008), although the exact number depends on the assumed efficiency in the conversion of gravitational energy to radiation.

The INTEGRAL and SWIFT missions explored the X-ray sky at energies above $10 \mathrm{keV}$ to provide the most unbiased samples of Compton-thick AGN in the local Universe. Owing to the limited imaging capabilities of these missions 
(coded-mask detectors), the flux limit probed is very high $\left(\sim 10^{-11} \mathrm{erg} \mathrm{cm}^{-2} \mathrm{~s}^{-1}\right)$, allowing only the detection of AGN at very low redshifts. These surveys did not detect large numbers of Compton-thick sources (e.g. Ajello et al. 2008; Tueller et al. 2008; Paltani et al. 2008; Winter et al. 2009; Burlon et al. 2011). The fraction of Compton-thick AGN in these surveys does not exceed a few percent of the total AGN population. In contrast, optical and mid-IR surveys yield Compton-thick AGN fractions of between 10\% and 20\% (Akylas \& Georgantopoulos 2009; Brightman \& Nandra 2011a). As Burlon et al. (2011) point out, it is possible that even these ultra-hard surveys are biased against the most heavily obscured $\left(>2 \times 10^{24} \mathrm{~cm}^{-2}\right)$ Comptonthick sources.

At higher redshifts, a number of efforts have been made to identify Compton-thick AGN by means of either X-ray hardness ratios (Brunner et al. 2008; Gilli et al. 2011) or X-ray spectroscopy (Tozzi et al. 2006; Georgantopoulos et al. 2009; Comastri et al. 2011; Feruglio et al. 2011) in the deepest Chandra and XMM-Newton observations. In particular, Comastri et al. (2011), provided the most direct X-ray spectroscopic evidence yet for the presence of Compton-thick nuclei at high redshift, reliably identifying two Compton-thick AGN at $z=1.536$ and $z=3.700$.

The mid-IR data provide an alternative way to detect heavily obscured systems. This is because the radiation absorbed by circumnuclear dust is re-emitted in the IR part of the spectrum (Soifer et al. 2008), rendering heavily obscured AGN copious mid-IR emitters. In these systems, the $2-10 \mathrm{keV} X$-ray emission can be diminished by almost two orders of magnitude (e.g. Matt et al. 2004), while at the same time the isotropic mid-IR emission remains largely unattenuated. Daddi et al. (2007), Fiore et al. (2008), Georgantopoulos et al. (2008), Treister et al. (2009b), Eckart et al. (2010), Donley et al. (2010), Luo et al. (2011), Alexander et al. (2011) propose that a fraction of the infrared excess, $24 \mu \mathrm{m}$-bright sources that are undetected in X-rays are associated with Compton-thick AGN. Their main argument is again the very low X-ray to IR luminosity ratio which is indicative of significant amounts of absorption (but see Georgakakis et al. 2010; Georgantopoulos et al. 2011).

The detection of a low $L_{\mathrm{X}} / L_{6 \mu \mathrm{m}}$ luminosity ratio has been used as the main instrument for the detection of faint Comptonthick AGN which remain undetected in X-rays (e.g. Alexander et al. 2008; Goulding et al. 2011). This is because the $6 \mu \mathrm{m} \mathrm{lu}-$ minosity is an excellent proxy of the AGN power, as it should be dominated by very hot dust heated by the AGN (e.g. Lutz et al. 2004; Maiolino et al. 2007). At these wavelengths, the contribution of the stellar light and colder dust heated by young stars should be minimal. Yaqoob \& Murphy (2011) criticise the above results claiming that the $L_{\mathrm{X}} / L_{6} \mu \mathrm{m}$ ratio is not a robust indicator of Compton-thick obscuration. They perform Monte Carlo X-ray spectral simulations of heavily obscured AGN. They caution that Compton-thick AGN may have the same X-ray to mid-IR luminosity ratio as heavily obscured AGN which have a column density of a few times $10^{23} \mathrm{~cm}^{-2}$, depending on the exact value of the covering fraction and photon index.

In this paper, we attempt to assess the efficiency of the $L_{\mathrm{X}} / L_{6 \mu \mathrm{m}}$ ratio method, i.e. to estimate the percentage of Compton-thick AGN among the low $L_{X} / L_{6 \mu \mathrm{m}}$ sources, but also to evaluate whether there are any bona fide Compton-thick AGN with substantially high $L_{X} / L_{6 \mu \mathrm{m}}$ ratios. First, we use a sample of local AGN, the IRAS $12 \mu \mathrm{m}$ sample of Rush et al. (1993), to assess the reliability and effectiveness of the $L_{\mathrm{X}} / L_{6 \mu \mathrm{m}}$ ratio selection (Sect. 3.1). For this sample excellent quality X-ray spectroscopic observations are available (Brightman \& Nandra 2011a), and hence we know a priori which objects are Compton-thick. Second, we select candidate Compton-thick AGN among the low $L_{\mathrm{X}} / L_{6 \mu \mathrm{m}} \mathrm{AGN}$ in the CDFS (Sect. 3.2). We present a detailed X-ray spectral analysis of these sources, using the most sensitive X-ray data ever obtained, the $4 \mathrm{Ms}$ Chandra data, combined with the $3 \mathrm{Ms} X M M-N e w t o n$ data. Our aim is to detect unambiguous signs of Compton-thick obscuration, such as direct detection of a large column density, or a flat spectral index, or a large equivalent-width (hereafter $E W$ ) $\mathrm{Fe} \mathrm{K} \alpha$ line. We adopt $H_{0}=75 \mathrm{~km} \mathrm{~s}^{-1} \mathrm{Mpc}^{-1}, \Omega_{\mathrm{M}}=0.3$, and $\Omega_{\Lambda}=0.7$ throughout the paper.

\section{Data}

\subsection{The IRAS $12 \mu \mathrm{m}$ sample}

Here, we describe the available X-ray (XMM-Newton) and midIR data available for the IRAS sample.

\subsubsection{XMM-Newton data}

The extended IRAS $12 \mu \mathrm{m}$ galaxy survey (Rush et al. 1993) contains 893 MIR selected local galaxies. A high fraction of these galaxies, are AGN (over 10\%) according to optical spectroscopy. The sample is taken from the IRAS Faint Source Catalogue, version 2 (FSC-2) and imposes a flux limit of $0.22 \mathrm{Jy}$, including only sources with a rising flux density from 12 to 60 microns (to minimise the contamination by stars) and with a Galactic latitude of $|b|>25^{\circ}$. There are XMM-Newton observations available for 126 galaxies from the $12 \mu \mathrm{m}$ sample.

\subsubsection{Mid-IR data}

For the local sources, we used photometry from the NASA Extragalactic Database (NED). The most commonly used datasets are the 2MASS survey (Skrutskie et al. 2006) for the near-IR, and IRAS, ISO, and Spitzer catalogues for the near-IR, mid-IR, and far-IR. We used only broad-band photometry datapoints, and avoided measurements made with apertures smaller than the apparent sizes of the sources. The number of data-points used range from seven (IRAS 07599+6508) up to 33 (Mrk 231).

\subsection{CDFS}

Next, we describe the X-ray (XMM-Newton and Chandra) as well as the mid-IR (Spitzer) data available in the CDFS.

\subsubsection{Chandra}

The CDFS 4 Ms observations consist of 53 pointings obtained in the years 2000 (1 Ms), 2007 (1 Ms), and 2010 (2 Ms). The analysis of the first $1 \mathrm{Ms}$ data is presented in Giacconi et al. (2002) and Alexander et al. (2003), while the analysis of the 23 observations obtained up to 2007 is presented in Luo et al. (2008). These authors detected $462 \mathrm{X}$-ray sources in the $2 \mathrm{Ms}$ data. The average aim point is $\alpha=03^{\mathrm{h}} 32^{\mathrm{m}} 28^{\mathrm{s}} .8, \delta=-27^{\circ} 48^{\prime} 23^{\prime \prime}$ (J2000). The observations cover an area of about $440 \mathrm{arcmin}^{2}$. The present CDFS $4 \mathrm{Ms}$ survey reaches a sensitivity limit of $\sim 0.7 \times 10^{-16} \mathrm{erg} \mathrm{cm}^{-2} \mathrm{~s}^{-1}$ and $1 \times 10^{-17} \mathrm{erg} \mathrm{cm}^{-2} \mathrm{~s}^{-1}$ in the hard $(2-8 \mathrm{keV})$ and soft $(0.5-2 \mathrm{keV})$ band, respectively (Xue et al. 2011). The Galactic column density towards the CDFS is $0.9 \times$ $10^{20} \mathrm{~cm}^{-2}$ (Dickey \& Lockman 1990). 


\subsubsection{XMM-Newton}

The CDFS area was surveyed with XMM-Newton during different epochs spread over almost nine years. The data presented in this paper combine the observations awarded to our project in AO7 and AO8 (Comastri et al. 2011). These observations are performed in four different epochs between July 2008 and March 2010, while the archival data were acquired in the period July 2001-January 2002. The total exposure time, after the removal of background flares, is $\approx 2.82 \mathrm{Ms}$ for the two MOS and $\approx 2.45 \mathrm{Ms}$ for the PN detectors. An extended and detailed description of the full data set, including data analysis and reduction and the X-ray catalogue will be published in Ranalli et al. (in prep.).

\subsubsection{Mid-IR}

The central regions of the CDFS were observed in the mid-IR by the Spitzer mission as part of the Great Observatory Origin Deep Survey (GOODS). These observations cover areas of about $10 \times 16.5 \mathrm{arcmin}^{2}$ in both fields using the IRAC $(3.6,4.5,5.8$, and $8.0 \mu \mathrm{m})$ and the MIPS (24 and $70 \mu \mathrm{m})$ instruments on board Spitzer. We construct a uniform dataset of infrared fluxes by matching the $2 \mathrm{Ms}$ X-ray catalogue of Luo et al. (2008) with the IRAC catalogue from the SIMPLE survey (Damen et al. 2011), which covers the total area of the CDFS. We find counterparts for 446 out of the 462 sources. We also use the FIDEL survey of the CDFS (see Magnelli et al. 2009) to get photometric information at longer wavelengths $(24 \mu \mathrm{m}$ and $70 \mu \mathrm{m})$. There are 161 sources with mid-IR data in the four IRAC bands, 210 sources with five bands (IRAC + MIPS $24 \mu \mathrm{m}$ ) and finally 69 sources with mid-IR data in six bands (IRAC + MIPS, $24 \mu \mathrm{m}$ and $70 \mu \mathrm{m}$ ). For the remaining six sources we only have information in fewer than four IRAC bands. For these sources, we do not derive spectral energy distribution (SED) fits.

\section{Analysis}

\section{1. $12 \mu \mathrm{m}$ sample}

For the local sample, we used the XMM-Newton spectroscopic information presented in Brightman \& Nandra (2011a). These authors provided X-ray spectral fits with particular emphasis on revealing the Compton-thick sources. There are 60 high X-ray luminosity sources (i.e. with intrinsic $L_{\mathrm{X}}>10^{42} \mathrm{erg} \mathrm{s}^{-1}$ ) among the 126 sources, with XMM-Newton observations presented in Brightman \& Nandra (2011a). These 60 sources were classified as bona fide AGN on the basis of their high X-ray luminosity alone. The X-ray luminosity classification is based on the fact that the most X-ray luminous star forming galaxies (e.g. NGC 3256; Moran et al. 1999), have a 2-10 keV luminosity below $10^{42} \mathrm{erg} \mathrm{s}^{-1}$, without showing evidence of AGN activity. Among these 60 sources, ten AGN display X-ray spectral evidence of Compton-thick obscuration according to Brightman \& Nandra (2011a). That is their X-ray spectra contain either a) large $E W \mathrm{FeK}_{\alpha}$ lines, or b) flat spectral indices indicative of a reflection component, or c) direct detections of an absorption turn-over with $N_{\mathrm{H}}>10^{24} \mathrm{~cm}^{-2}$.

To construct the $L_{X} / L_{6 \mu \mathrm{m}}$ diagram, we consider the X-ray luminosities (uncorrected for absorption) of the AGN in the $12 \mu \mathrm{m}$ sample derived from the XMM-Newton fluxes of Brightman \& Nandra (2011a). The $6 \mu \mathrm{m}$ IR flux was estimated using SED fits (see Sect. 3.3).

\subsection{CDFS}

\subsubsection{Chandra spectra}

For the CDFS sample, we derived X-ray spectra for the sources in the Luo et al. (2008) catalogue using the 4 Ms data (Xue et al. 2011).

We used the SPECEXTRACT script in the CIAO v4.2 software package to extract the spectra of all 462 X-ray sources in our sample. The extraction radius varies between 2 and 4 arcsec with increasing off-axis angle. At low off-axis angles ( $<4$ arcmin), this area encircles $90 \%$ of the light at an energy of $1.5 \mathrm{keV}$. The same script extracts response and auxiliary files. The addition of the spectral files was performed with the FTOOL task MATHPHA. To add the response and auxiliary files, we used the FTOOL ADDRMF, and ADDARF tasks respectively, weighting according to the number of photons in each spectrum.

We fit the spectra using an absorbed power-law model PLCABS (Yaqoob 1997), which properly takes into account Compton scattering up to column densities of $N_{\mathrm{H}} \sim 10^{25} \mathrm{~cm}^{-2}$. We used the XSPEC v12.5 software package for the spectral fits (Arnaud 1996). We used the C-statistic technique (Cash 1979), which had been specifically developed to extract spectral information from data of low signal-to-noise ratio. We derived accurate $\mathrm{X}$-ray luminosities from the $\mathrm{X}$-ray spectral fits. This is the most reliable method for deriving X-ray luminosities. In contrast, the derivation of the X-ray luminosity on the basis of the $\mathrm{X}$-ray count rate and an average photon index can substantially distort the $L_{X} / L_{6 \mu \mathrm{m}}$ relation.

\subsubsection{XMM-Newton spectra}

For each individual XMM-Newton orbit, source counts were collected from a circular region of 12 arcsec radius, centered on the source position. Local background data were taken from nearby regions, separately for the PN, MOS1, and MOS2 detectors where no source had been found in the full exposure image. The area of the background region was defined to have 20 arcsec radius. The spectral data from individual exposures were summed for the source and background, respectively, and a background subtraction was made assuming a common scaling factor for the source/background geometrical areas. Both PN and MOS spectra are extracted in the $0.5-8 \mathrm{keV}$ energy area. MOS1 and MOS2 spectra are summed using the FTOOLS MATHPHA task. Response and effective area files were computed by averaging the individual files using the FTOOLS ADDRMF and ADDARF tasks.

\subsection{Spectral energy distributions}

We constructed an SED to help us ascertain the predominant powering mechanism (AGN or star formation) in the mid-IR part of the spectrum. The SEDs were also used to obtain an accurate estimate of the $6 \mu \mathrm{m}$ infrared luminosities. We used a $\chi^{2}$ minimisation technique to determine the optimal combinations of host galaxy and pure AGN SED templates reproducing the broad-band spectra of our objects. The starburst templates we used, were those from the SWIRE template library of Polletta et al. (2007), which is a compilation of observed SEDs of nearby galaxies (M 82, NGC 6090, Arp 220, IRAS 20547, IRAS 22491). In addition, we used the templates of Chary \& Elbaz (2001). The AGN templates we used come from Silva et al. (2004), who combine nuclear SEDs of Seyferts with a range of absorption column densities. We also constructed a 

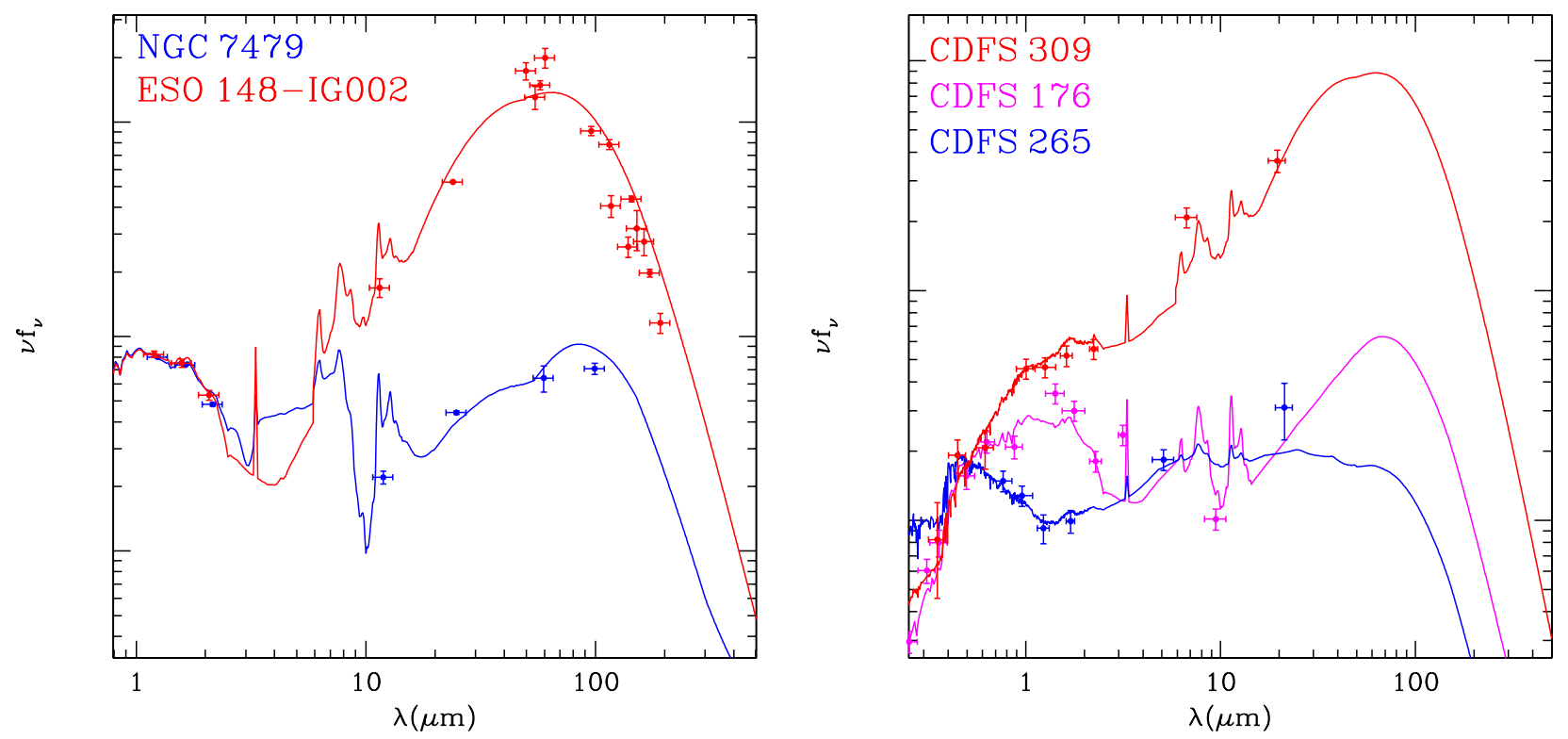

Fig. 1. Left panel: the SED of the Compton-thick sources NGC 7479 and ESO148-IG002 from the $12 \mu \mathrm{m}$ sample. Right panel: the SED of the two Compton-thick sources in Comastri et al. (2011) compared to that of the candidate Compton-thick source CDFS-309 in Feruglio et al. (2011). The SEDs have been normalised to have the same flux at $0.5 \mu \mathrm{m}$.

sample of AGN templates using the type-1 QSO SED from Richards et al. (2006) and applying dust absorption following Rosenthal et al. (2000), to account for the $9.7 \mu \mathrm{m}$ absorption feature. In Fig. 1, we show an example SED of Compton-thick sources in both the high and low-redshift Universe.

\section{Results}

\subsection{The IRAS $12 \mu \mathrm{m}$ sample}

We present the $L_{X} / L_{6 \mu \mathrm{m}}$ diagram for the nearby sources in Fig. 2. The X-ray luminosity is uncorrected for obscuration, while the $6 \mu \mathrm{m}$ luminosity includes both the torus and the starformation component (see discussion). The lines denote various Compton-thick AGN areas. These were derived in the following fashion. We adopted the average $L_{\mathrm{X}} / L_{6 \mu \mathrm{m}}$ luminosity-dependent AGN relation of Fiore et al. (2009) derived in the COSMOS field. We then scaled this relation down by a factor of 30 . This is an approximation to the average reflected luminosity relative to the intrinsic emission from the backside of the torus in the $2-10 \mathrm{keV}$ band in reflection-dominated AGN (e.g. Comastri 2004). Alternatively, $3 \%$ is the relation between the absorbed and unabsorbed $2-10 \mathrm{keV}$ luminosity for a column density of $1.5 \times 10^{24} \mathrm{~cm}^{-2}$ and a power-law spectrum of $\Gamma=1.8$. We show an alternative scenario, where the relation of Fiore et al. (2009) is scaled for only $1 \%$ reflected emission (Vignali et al. 2004). We also give the $L_{X} / L_{6} \mu \mathrm{m}$ relation derived by Lutz et al. (2004) in the local Universe, together with its associated $1 \sigma$ uncertainty scaled down again by $3 \%$ (shaded area). Throughout the paper, we used only the Fiore et al. (2009) $3 \%$ reflection limit to assess the number of Compton-thick sources. We see that most (ten out of eleven) of the sources classified as Compton-thick on the basis of X-ray spectroscopy lie below the Compton-thick lines. However, we see that many more sources (twelve) that are not Compton-thick, according to the XMM-Newton X-ray spectroscopic diagnostics, would be classified as candidate Comptonthick AGN on the basis of the low $L_{X} / L_{6 \mu}$ m ratio. All these are presented in Table 1. It is not unlikely that some of the low $L_{\mathrm{X}} / L_{6 \mu \mathrm{m}}$ sources are indeed Compton-thick sources, but
$X M M-N e w t o n$ failed to identify them owing to the limited quality of the photon statistics. At least two sources NGC 424, (Iwasawa et al. 2001; Burlon et al. 2011) and UGC 8058 (Mrk 231, Braito et al. 2004), are Compton-thick AGN on the basis of SWIFT and BeppoSAX spectroscopy.

\subsection{CDFS}

We excluded all sources with an intrinsic X-ray luminosity of $L_{\mathrm{X}}<10^{42} \mathrm{erg} \mathrm{s}^{-1}$, as many of these could be associated with normal galaxies. We found that 283 sources have luminosities above this level. The $L_{X} / L_{6 \mu \mathrm{m}}$ diagram is shown in Fig. 3. Nine sources would be characterised as Compton-thick on the basis of their low $L_{X} / L_{6 \mu \mathrm{m}}$ ratio (see Table 2). In Table 2, we present the Chandra spectral fit results. Throughout our paper, we use the Luo et al. (2008) identification numbers. Four low $-L_{\mathrm{X}} / L_{6} \mu \mathrm{m}$ sources could be classified as Compton-thick: CDFS-309, CDFS-95, CDFS-197, and CDFS-398. CDFS-309 was discussed in detail in Feruglio et al. (2011) and found to be a Compton-thick source on the basis of an $\mathrm{Fe} \mathrm{K} \alpha$ line with a large $E W$. Owing to the limited photon-statistics we cannot tell whether the source has a flat spectrum with $\Gamma \approx 0.6$ or is obscured with $N_{\mathrm{H}} \sim 3 \times 10^{23} \mathrm{~cm}^{-2}$. However, the addition of an $\mathrm{FeK} \alpha$ line improves the statistic by $\Delta C \approx 20$ yielding an $E W$ of $3.6 \pm 1.8 \mathrm{keV}$. CDFS-95 and CDFS-197 have column densities somewhat below the Compton-thick limit $\left(\sim 9 \times 10^{23} \mathrm{~cm}^{-2}\right)$, but are nevertheless consistent with being Compton-thick within the errors. However, for both sources there are photometric redshifts only available and hence the column density estimates are uncertain.

Only two low $-L_{\mathrm{X}} / L_{6 \mu \mathrm{m}}$ sources have fluxes $f_{2-10 \mathrm{keV}}>$ $10^{-15} \mathrm{erg} \mathrm{cm}^{-2} \mathrm{~s}^{-1}$. These sources have also been detected by $X M M-N e w t o n$, allowing a spectral fit with both the photon index and the column density considered as free parameters. These are CDFS-9 and CDFS-398. In Table 3, we present the spectral fit results for these two sources. The spectrum of CDFS-9 is highly absorbed with a column density of $\sim 10^{23} \mathrm{~cm}^{-2}$, but it is certainly not a Compton-thick source. From Table 3, we see 
I. Georgantopoulos et al.: X-ray to $6 \mu \mathrm{m}$ luminosity ratio

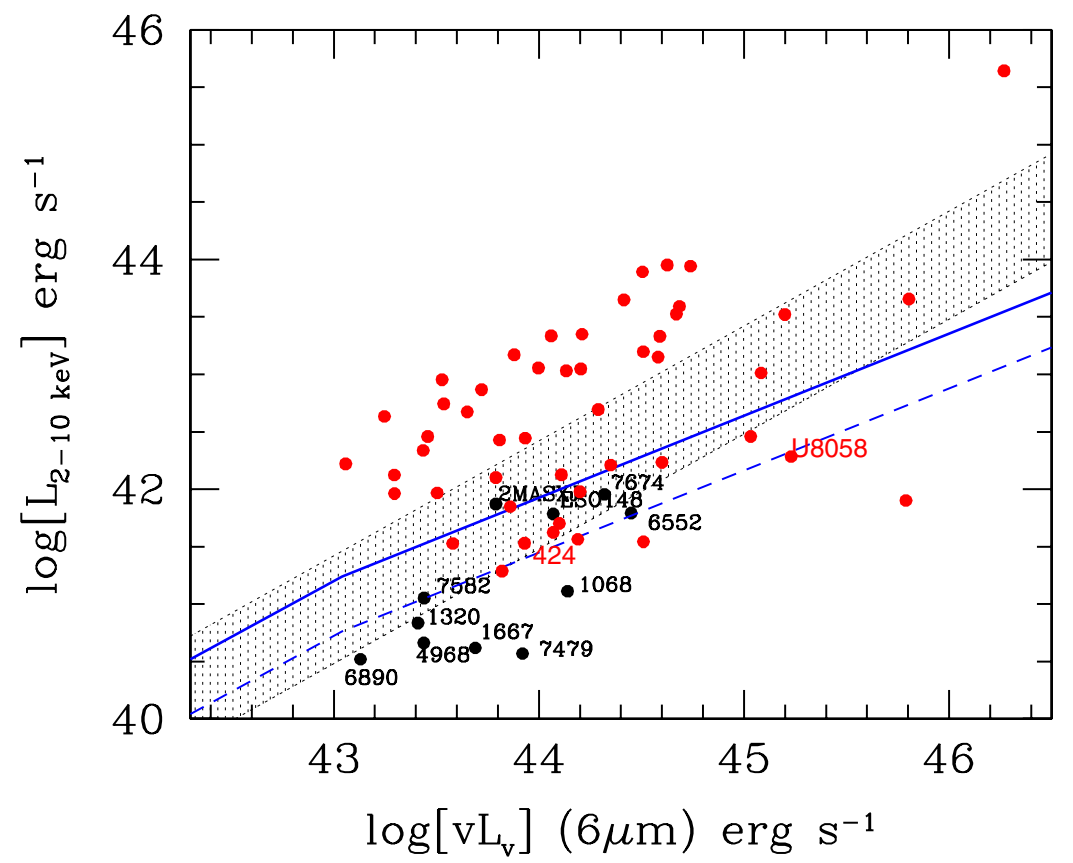

Fig. 2. Rest-frame $L_{\mathrm{X}}$ versus (vs.) $L_{6 \mu \mathrm{m}}$ luminosity diagram for the unambiguous AGN i.e. those with intrinsic X-ray $2-10 \mathrm{keV}$ luminosity $L_{\mathrm{X}}>10^{42} \mathrm{erg} \mathrm{s}^{-1}$. The X-ray luminosity is uncorrected for absorption, while the mid-IR luminosity includes both the torus and the star-formation component. The black and the red circles correspond to the Compton-thick and Compton-thin sources in the AGN $\left(L_{\mathrm{X}}>10^{42} \mathrm{erg} \mathrm{s}^{-1}\right)$ sample of Brightman \& Nandra (2011a). The shaded area denotes the Compton-thick regime based on the average $L_{\mathrm{X}} / L_{6 \mu \mathrm{m}}$ relation (corrected for obscuration) found for local AGN by Lutz et al. (2004) scaled down assuming a 3\% fraction of reflected emission from the backside of the obscuring screen in the $2-10 \mathrm{keV}$ band. The dispersion in this relation is $\approx 0.7 \mathrm{dex}$ for type- $2 \mathrm{AGN}$. The blue lines show the luminosity-dependent average relation for Compton-thick AGN based on the COSMOS AGN assuming a 3\% (solid-line) and a 1\% (dashed-line) fraction of reflected emission. Only one Compton-thick source (2MASX J15504152) lies above the nominal Compton-thick regime as defined by the Fiore et al. (2009) average relation assuming a $3 \%$ reflection.

that the Chandra spectrum of CDFS-398 is flat $(\Gamma \approx 0.8 \pm 0.3)$. Since the source was detected by XMM-Newton, data with good photon statistics are available allowing a more complex fit to the spectrum. We considered two spectral models to fit its X-ray spectrum, (1) an absorbed power-law spectrum with the addition of an FeK $\alpha$ line, (plcabs+zga); and (2) a reflection-dominated model with an $\mathrm{FeK} \alpha$ line, pexrav+zga. The spectral fits to the combined Chandra and XMM-Newton data are given in Table 3. This source displays a very flat spectrum $\Gamma<1.2$ at the $90 \%$ confidence level. This flat spectrum is characteristic of reflectiondominated Compton-thick sources (Matt et al. 2004). The restframe $E W$ of the $\mathrm{Fe} \mathrm{K} \alpha$ line is $0.68_{-0.44}^{+0.66} \mathrm{keV}$, which is again consistent with Compton-thick emission (Fukazawa et al. 2011). The X-ray spectrum is presented in Fig. 4.

For clarity, we summarise the numbers of $L_{X} / L_{6 \mu \mathrm{m}}$ sources in the various subsamples given in this section in Table 5.

\section{Discussion}

\subsection{The local sample}

We have considered 60 bona fide AGN, with luminosities $L_{2-10 \mathrm{keV}}>10^{42} \mathrm{erg} \mathrm{s}^{-1}$ from the $12 \mu \mathrm{m}$ IRAS sample. $X M M-N e w t o n$ observations of these sources are presented in Brightman \& Nandra (2011a) and Brightman \& Nandra (2011b). Twenty-two of these AGN have low $L_{X} / L_{6 \mu \mathrm{m}}$ ratios. In particular, their X-ray luminosity is lower than the $3 \%$ of the average AGN luminosity-dependent $L_{X} / L_{6 \mu \mathrm{m}}$ relation derived by Fiore et al. (2009). This is assumed to be the fraction of the reflected $2-10 \mathrm{keV}$ emission from the backside of the torus, relative to the intrinsic $2-10 \mathrm{keV}$ luminosity. Although this value of reflected emission is often observed in the local Universe (e.g. Comastri 2004), it should only be considered as a rough approximation. For example, it is likely that in some cases it could be even smaller (Ueda et al. 2007; Comastri et al. 2010). It is noteworthy that the $L_{X} / L_{6 \mu}$ method can retrieve the vast majority (ten out of eleven) of the Compton-thick AGN found by Brightman \& Nandra (2011a). This demonstrates that the empirical limit adopted above is efficient for the selection of Compton-thick sources. The X-ray spectroscopy reveals that ten out of the 22 low $L_{\mathrm{X}} / L_{6 \mu \mathrm{m}}$ AGN are Compton-thick AGN following the classification of Brightman \& Nandra (2011a). Most of the remaining twelve sources are heavily obscured having $N_{\mathrm{H}}>10^{23} \mathrm{~cm}^{-2}$, but these are not formally Compton-thick, at least according to the XMM-Newton spectroscopy. This suggests that the low $L_{X} / L_{6 \mu}$ m ratio technique provides roughly a $45 \%$ success rate in detecting Compton-thick sources in the local Universe. Interestingly, the level of contamination is not extremely sensitive to the exact value of the adopted $L_{X} / L_{6 \mu \mathrm{m}}$ upper limit. For example, from Fig. 2, we can easily estimate that by lowering the adopted threshold e.g. by as much as a factor of three (i.e. adopting a $1 \%$ reflection fraction), we end up with eleven sources, six of which are classified as Compton-thick by Brightman \& Nandra (2011a). The number of Compton-thick sources rises to seven after taking into account UGC8058 which is a Compton-thick source according to BeppoSAX spectroscopy (Braito et al. 2004) as noted above.

One important parameter that is often ignored is the fraction of the star-formation contribution to the $6 \mu \mathrm{m}$ emission. In most cases $(15 / 23)$, the torus component is the dominant contributor to the $6 \mu \mathrm{m}$ luminosity. In the remaining cases, the 
Table 1. Candidate Compton-thick sources in the local sample based on the $L_{\mathrm{X}} / L_{6 \mu \mathrm{m}}$ ratio.

\begin{tabular}{lccccccc}
\hline \hline Name & $z$ & $L_{\mathrm{X}}$ & $L_{6 \mu \mathrm{m}}$ & Fraction & $\mathrm{CT}$ & $N_{\mathrm{H}}$ & $E W$ \\
$(1)$ & $(2)$ & $(3)$ & $(4)$ & $(5)$ & $(6)$ & $(7)$ & $(8)$ \\
\hline NGC 17 & 0.0196 & 41.29 & 43.82 & 0 & - & $47_{-21.2}^{+30.4}$ & 0.440 \\
NGC 424 & 0.0118 & 41.60 & 43.93 & 1 & - & $23.6_{-15.8}^{+44.7}$ & $<0.024$ \\
NGC 1068 & 0.0380 & 41.11 & 44.14 & 0.74 & $\mathrm{~b}$ & - & - \\
NGC 1194 & 0.0136 & 41.53 & 43.58 & 0.91 & - & $67.8_{-16.4}^{+18.3}$ & $<0.059$ \\
NGC 1320 & 0.0890 & 40.83 & 43.41 & 0.83 & $\mathrm{a}$ & $204_{-99}^{+91}$ & 0.082 \\
NGC 1667 & 0.0152 & 40.62 & 43.69 & 0 & $\mathrm{a}$ & $271_{-251}^{+5730}$ & 0.391 \\
F 07599+6508 & 0.1488 & 41.90 & 45.79 & 0.96 & - & - & 0.027 \\
UGC 5101 & 0.0394 & 41.70 & 44.10 & 0 & - & $49.6_{-18.2}^{+25.4}$ & 0.229 \\
Mrk 231 & 0.0422 & 42.28 & 45.23 & 0.95 & - & $7.1_{-1.4}^{+1.8}$ & 0.011 \\
NGC 4968 & 0.0099 & 40.66 & 43.44 & 0.92 & $\mathrm{a}$ & $300_{-123}^{+2230}$ & 0.172 \\
NGC 5256 & 0.0279 & 41.62 & 44.07 & 0 & - & $17.5_{-3.8}^{+5.6}$ & 0.608 \\
Mrk 273 & 0.0378 & 41.98 & 44.20 & 0 & - & $59.7_{12.8}^{+17.1}$ & 0.192 \\
Mrk 463 & 0.0504 & 42.46 & 45.03 & 0.98 & - & $38.7_{-5.7}^{+9.2}$ & $<0.008$ \\
Mrk 848 & 0.0402 & 41.54 & 44.51 & 0.79 & - & $85.9_{-62.2}^{+2030}$ & - \\
† 2MASX J15504152 & 0.0303 & 41.87 & 43.79 & 0.48 & $\mathrm{c}$ & $0.36_{-0.28}^{+0.54}$ & - \\
NGC 6552 & 0.0265 & 41.79 & 44.55 & 0.95 & $\mathrm{~b}$ & - & - \\
F 19254-7245 & 0.0617 & 42.23 & 44.60 & 0.88 & - & $38.1_{-21.7}^{+39.2}$ & 0.064 \\
NGC 6890 & 0.0081 & 40.52 & 43.13 & 0.24 & $\mathrm{c}$ & $0.11_{-0.09}^{+0.20}$ & 0.237 \\
NGC 7479 & 0.0079 & 40.57 & 43.92 & 0.70 & $\mathrm{a}$ & $201_{-122}^{+493}$ & - \\
NGC 7582 & 0.0053 & 41.05 & 43.44 & 0 & $\mathrm{c}$ & 0.04 & - \\
NGC 7674 & 0.0289 & 41.95 & 44.32 & 0.58 & $\mathrm{c}$ & - & 0.132 \\
ESO 286-IG019 & 0.0430 & 41.56 & 44.19 & 0.85 & - & $48.7_{-35.2}^{+258}$ & - \\
ESO 148-IG002 & 0.0446 & 41.78 & 44.07 & 0 & $\mathrm{c}$ & - & - \\
\hline & & & & & & & \\
\hline
\end{tabular}

Notes. The columns are: (1) name; (2) spectroscopic redshift; (3) logarithm of luminosity in the 2-10 keV band uncorrected for absorption in units of $\mathrm{erg} \mathrm{s}^{-1}$; (4) logarithm of $6 \mu \mathrm{m}$ luminosity in units of $\mathrm{erg} \mathrm{s}^{-1}$; (5) fraction of the AGN contribution to the $6 \mu \mathrm{m}$ luminosity; (6) compton-thick classification from Brightman \& Nandra (2011a) based on: a: direct measurement of the column density; b: large $E W$ of Fe K $\alpha$ line; c: large reflection component; (7) $N_{\mathrm{H}}$ column density in units of $10^{22} \mathrm{~cm}^{-2}$ from Brightman \& Nandra (2011a); (8) $E W$ of the $6.2 \mu \mathrm{m}$ PAH in units of $\mu \mathrm{m}$ from Wu et al. (2009); ${ }^{(\dagger)}$ source not in the low- $L_{\mathrm{X}} / L_{6 \mu \mathrm{m}}$ sample.

Table 2. Chandra spectral fits and $6 \mu \mathrm{m}$ luminosities of the low $L_{\mathrm{X}} / L_{6 \mu \mathrm{m}}$ sources in the CDFS.

\begin{tabular}{|c|c|c|c|c|c|c|c|c|c|c|c|c|c|}
\hline $\begin{array}{l}\text { ID } \\
(1)\end{array}$ & $\begin{array}{c}z \\
(2)\end{array}$ & $\begin{array}{l}N_{\mathrm{H}} \\
(3)\end{array}$ & $\begin{array}{c}\Gamma \\
(4)\end{array}$ & $\begin{array}{l}\text { C-stat } \\
\text { (5) }\end{array}$ & $\begin{array}{l}f_{2-10} \\
(6)\end{array}$ & $\begin{array}{l}L_{\mathrm{X}} \\
(7)\end{array}$ & $\begin{array}{c}L_{6 \mu \mathrm{m}}^{\mathrm{tot}} \\
(8)\end{array}$ & $\begin{array}{c}\text { Fraction } \\
\text { (9) }\end{array}$ & $\begin{array}{l}N_{\mathrm{H}} \\
(10)\end{array}$ & $\begin{array}{l}\text { C-stat } \\
\text { (11) }\end{array}$ & $\begin{array}{c}\Gamma \\
(12)\end{array}$ & $\begin{array}{l}\text { C-stat } \\
\text { (13) }\end{array}$ & $\begin{array}{c}\text { Counts } \\
\text { (14) }\end{array}$ \\
\hline 9 & 1.615 & $10.8_{-5.0}^{+4.6}$ & $2.53_{-0.84}^{+0.73}$ & 438 & 1.5 & 1.8 & 11.77 & 0.92 & $8.7_{-3}^{+4}$ & 439 & $0.88_{-0.25}^{+0.15}$ & 456 & 130 \\
\hline 95 & $5.73 \dagger$ & $83.4_{-7.6}^{+8.7}$ & $1.80_{-0.85}^{+1.10}$ & 505 & 1.0 & 2.7 & 12.96 & 1.0 & $94_{-38}^{+29}$ & 505 & $0.94_{-0.35}^{+0.26}$ & 508 & 137 \\
\hline 197 & $3.64 \dagger$ & $126_{-125}^{+365}$ & $2.65_{-2.4}^{+5.3}$ & 474 & 0.46 & 0.59 & 11.74 & 0.97 & $86_{-50}^{+39}$ & 437 & $0.55_{-0.65}^{+0.50}$ & 472 & 37 \\
\hline 282 & 2.223 & - & - & - & 0.14 & 0.38 & 11.95 & 0.87 & $<1.2$ & 477 & $2.98_{-0.96}^{+1.14}$ & 472 & 24 \\
\hline 293 & 3.10 & - & - & - & 0.15 & 0.65 & 11.75 & 0.96 & $16.2_{-15}^{+21}$ & 452 & $1.09_{-0.53}^{+0.75}$ & 455.5 & 22 \\
\hline 309 & 2.579 & - & - & - & 0.35 & 1.5 & 11.61 & 0.81 & $30_{-14}^{+24}$ & 497 & $0.60_{-0.57}^{+0.57}$ & 498 & 52 \\
\hline 382 & 0.664 & $26.8_{-16.0}^{+29.5}$ & $2.27_{-0.65}^{+0.80}$ & 487 & 0.63 & 0.1 & 10.75 & 0.1 & $1.4_{-0.66}^{+0.77}$ & 488 & $1.46_{-0.44}^{+0.21}$ & 497 & 179 \\
\hline 398 & 1.222 & $3.2_{-3.0}^{+8.0}$ & $2.02_{-0.9}^{+0.47}$ & 483 & 1.50 & 0.54 & 11.77 & 0.88 & $3.6_{-1.8}^{+2.8}$ & 470 & $0.81_{-0.32}^{+0.30}$ & 465 & 94 \\
\hline 407 & 1.367 & $<3.9$ & $1.46_{-0.73}^{+1.32}$ & 464 & 0.13 & 0.12 & 10.83 & 0.35 & $<3.5$ & 464 & $1.48_{-0.72}^{+1.26}$ & 464 & 48 \\
\hline
\end{tabular}

Notes. The columns are: (1) ID in the catalogue of Luo et al. (2008); (2) redshift from Luo et al. (2010); two and three decimal digits denote photometric and spectroscopic redshift respectively; $\uparrow$ source not detected in the BVR bands Cardamone et al. (2010) or $K$ band Taylor et al. (2009) and thus the photometric redshift may be uncertain. Rafferty et al. (2011) gives a photometric redshift of $z=2.30$ and $z=3.02$ for the sources CDFS-197 an CDFS-293 respectively; (3) column density in units $10^{22} \mathrm{~cm}^{-2}$, (4) Photon index; (5) $C$-statistic value for 509 degrees of freedom for both $\Gamma$ and $N_{\mathrm{H}}$ free; (6) X-ray flux in the $2-10 \mathrm{keV}$ band in units of $10^{-15} \mathrm{erg} \mathrm{cm}^{-2} \mathrm{~s}^{-1}$, as estimated from the X-ray spectroscopy; (7) X-ray luminosity in the $2-10 \mathrm{keV}$ band (uncorrected for X-ray absorption) in units of $10^{43} \mathrm{erg} \mathrm{s}^{-1}$; (8) logarithm of total $6 \mu$ m luminosity (star-formation and torus) in units of solar luminosity; (9) fraction of torus contribution relative to total luminosity at $6 \mu \mathrm{m}$, according to SED decomposition; (10) column density in units $10^{22} \mathrm{~cm}^{-2}$ for photon index fixed to $\Gamma=1.8$; (11) C-statistic value for 510 degrees of freedom; (12) photon index for column density $N_{\mathrm{H}}=0 \mathrm{~cm}^{-2}$; (13) C-statistic value for 510 degrees of freedom; (14) net counts (background subtracted) in the $0.5-8 \mathrm{keV}$ band. All errors quoted refer to the $90 \%$ confidence level. 


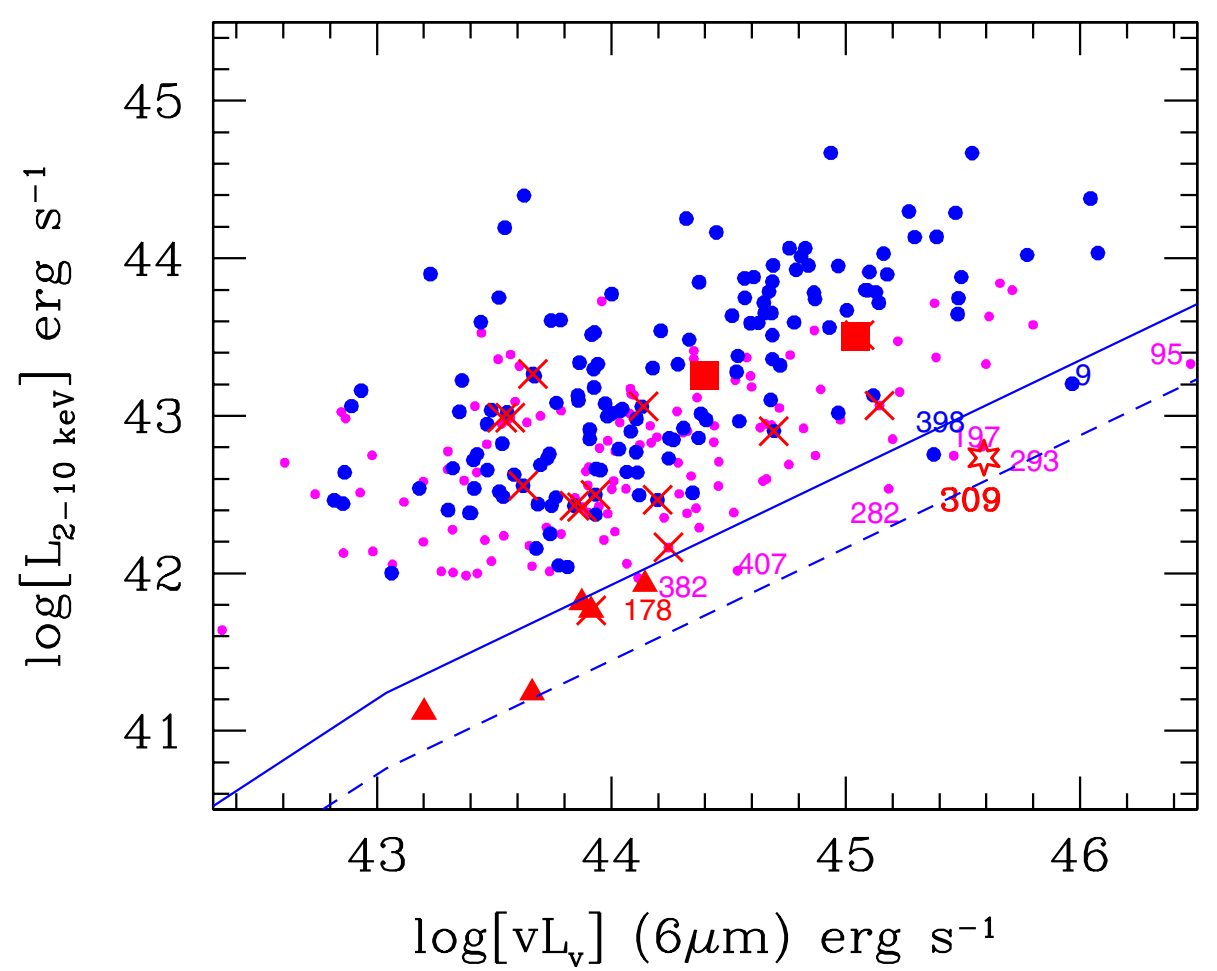

Fig. 3. Rest-frame (uncorrected for absorption) $L_{X}$ vs. $L_{6 \mu \mathrm{m}}$ luminosity diagram. The large filled circles (blue) correspond to the bright sources $\left(f_{2-10}>10^{-15} \mathrm{erg} \mathrm{cm}^{-2} \mathrm{~s}^{-1}\right)$, while the small circles (magenta) correspond to the fainter sources. The red squares correspond to the two bona fide Compton-thick AGN from the 3 Ms XMM observations (Comastri et al. 2011), while the red-star denotes the source CDFS-309, which was proposed as a Compton-thick AGN by Feruglio et al. (2011). The crosses refer to the 20 candidate Compton-thick sources of Tozzi et al. (2006) in the 1 Ms CDFS observations. These include both the reflected as well as the transmission-dominated sources; two sources not detected in Luo et al. (2008) and two more sources detected in fewer than four IRAC bands have been excluded. The red triangles correspond to the five low-luminosity sources $\left(L_{\mathrm{X}}<10^{42} \mathrm{erg} \mathrm{s}^{-1}\right)$ with flat spectra, given in Table 4. The solid line denotes the $L_{\mathrm{X}} / L_{6 \mu \mathrm{m}}$ average Compton-thick AGN relation; this has been derived from the average AGN relation of Fiore et al. (2009), scaled down to the expected Compton-thick AGN X-ray luminosity, assuming a 3\% fraction of torus reflected emission relative to the intrinsic $2-10 \mathrm{keV}$ luminosity. The dashed line shows the luminosity-dependent Compton-thick average $L_{\mathrm{X}} / L_{6 \mu \mathrm{m}}$ relation for an $1 \%$ torus-reflected emission.

Table 3. Chandra and XMM X-ray spectral fits of sources CDFS-9 and CDFS-398.

\begin{tabular}{|c|c|c|c|c|c|}
\hline $\begin{array}{l}\text { Model } \\
\text { (1) }\end{array}$ & $\begin{array}{l}\text { Mission } \\
(2)\end{array}$ & $\begin{array}{l}N_{\mathrm{H}} \\
(3) \\
\end{array}$ & $\begin{array}{c}\Gamma \\
(4) \\
\end{array}$ & $\begin{array}{l}E W \\
(5)\end{array}$ & $\begin{array}{c}\text { C-stat } \\
(6)\end{array}$ \\
\hline \multicolumn{6}{|c|}{ CDFS-9 } \\
\hline PL & XMM & $6.4_{-2.2}^{+3.1}$ & $1.43_{-0.28}^{+0.34}$ & - & $2892 / 2497$ \\
\hline PL + Line & XMM & $5.9_{-2.2}^{+2.9}$ & $1.41_{-0.28}^{+0.33}$ & $0.29 \pm 0.21$ & $2886 / 2496$ \\
\hline PL & Chandra + XMM & $8.2_{-2.6}^{+2.8}$ & $1.64_{-0.29}^{+0.29}$ & - & $3334 / 3008$ \\
\hline PL + Line & Chandra + XMM & $7.5_{-2.4}^{+2.8}$ & $1.58_{-0.29}^{+0.20}$ & $0.18_{-0.10}^{+0.10}$ & $3316 / 3007$ \\
\hline \multicolumn{6}{|c|}{ CDFS-398 } \\
\hline PL & XMM & $<0.68$ & $0.67_{-0.29}^{+0.26}$ & - & $2947 / 2499$ \\
\hline PL + Line & XMM & $<0.62$ & $0.68_{-0.27}^{+0.30}$ & $0.71 \pm 0.49$ & $2940 / 2498$ \\
\hline PL & Chandra + XMM & $<0.74$ & $0.86_{-0.26}^{+0.26}$ & - & $3405 / 3008$ \\
\hline PL + Line & Chandra + XMM & $<0.33$ & $0.78_{-0.21}^{+0.24}$ & $0.60 \pm 0.13$ & $3395 / 3007$ \\
\hline PEX + Line & Chandra + XMM & - & $2.68_{-0.3}^{+0.3}$ & $0.18_{-0.06}^{+0.06}$ & $3407 / 3010$ \\
\hline
\end{tabular}

Notes. The columns are: (1) model; (2) data used in the fits; (3) column density in units of $10^{22} \mathrm{~cm}^{-2}$; (4) photon index; (5) $E W$ (rest-fame) of the $\mathrm{FeK} \alpha$ line; (6) C-statistic value divided by degrees of freedom; all errors correspond to the $90 \%$ confidence level.

torus contribution to the $6 \mu \mathrm{m}$ emission is small i.e. $<30 \%$ (see Table 1). In the same table, we give the $E W$ of the PAH (polycyclic aromatic hydrocarbon) features at $6.2 \mu \mathrm{m}$ reported in $\mathrm{Wu}$ et al. (2009). A large $E W$ (usually above $0.3 \mu \mathrm{m}$ ) is considered to be an indicator of a strong starforming component. We see that sources that have a small torus contribution, as derived from the SED fit, generally have a large $E W$. The only marginal case is Mrk 273, which displays no torus in its SED, while the $E W$ of the $6.2 \mu \mathrm{m}$ PAH feature is $\approx 0.2 \mu \mathrm{m}$. For the sources that display a dominant star-forming contribution, one should be cautious in using the $L_{X} / L_{6} \mu \mathrm{m}$ ratio as a Compton-thick diagnostic, because the above ratio represents only a lower limit to the true value. In principle, in these cases one could use instead the torus $6 \mu \mathrm{m}$ luminosity. Nevertheless, 
Table 4. Low $L_{\mathrm{X}} / L_{6 \mu \mathrm{m}}$ and low intrinsic X-ray luminosity sources with flat spectra.

\begin{tabular}{|c|c|c|c|c|c|c|c|c|}
\hline $\begin{array}{l}\text { ID } \\
\text { (1) }\end{array}$ & $\begin{array}{c}z \\
(2)\end{array}$ & $\begin{array}{l}f_{2-10} \\
(3)\end{array}$ & $\begin{array}{l}L_{\mathrm{X}} \\
(4)\end{array}$ & $\begin{array}{c}L_{6 \mu \mathrm{m}}^{\mathrm{tot}} \\
(5)\end{array}$ & $\begin{array}{c}\Gamma \\
(6)\end{array}$ & $\begin{array}{c}\text { C-stat } \\
(7)\end{array}$ & $\begin{array}{l}N_{\mathrm{H}} \\
(8)\end{array}$ & $\begin{array}{c}\text { C-stat } \\
\text { (9) }\end{array}$ \\
\hline 96 & 0.310 & 0.76 & 41.23 & 10.07 & $0.83_{-0.41}^{+0.43}$ & 503 & $<0.53$ & 512 \\
\hline 159 & 0.664 & 0.78 & 41.81 & 10.28 & $0.39_{-0.71}^{+0.51}$ & 539 & $<0.24$ & 549 \\
\hline 166 & 0.340 & 0.54 & 41.12 & 9.61 & $0.33_{-0.77}^{+0.66}$ & 438 & $4.4_{-1.9}^{+4.7}$ & 440 \\
\hline 178 & 0.735 & 1.22 & 41.76 & 10.32 & $-0.81_{-2.0}^{+1.4}$ & 452 & $158_{-74}^{+83}$ & 449 \\
\hline 208 & 0.738 & 0.72 & 41.92 & 10.55 & $0.62_{-0.52}^{+0.52}$ & 488 & $<0.75$ & 496 \\
\hline
\end{tabular}

Notes. The columns are: (1) name in the catalogue of Luo et al. (2008); (2) spectroscopic redshift from Luo et al. (2010); (3) X-ray flux in the $2-10 \mathrm{keV}$ band in units of $10^{-15} \mathrm{erg} \mathrm{cm}^{-2} \mathrm{~s}^{-1}$, as estimated from the X-ray spectroscopy; (4) logarithm of the obscured X-ray luminosity in the $2-10 \mathrm{keV}$ band in units of $\mathrm{erg} \mathrm{s}^{-1}$; (5) logarithm of the total $6 \mu \mathrm{m}$ luminosity (star-formation and torus) in units of solar luminosity; (6) photon index $\Gamma$ for column density fixed to $N_{\mathrm{H}}=0$, as derived from the Chandra spectral fits; (7) corresponding C-statistic value; (8) column density in units of $10^{22} \mathrm{~cm}^{-2}$ for photon index fixed to $\Gamma=1.8$; (9) corresponding $\mathrm{C}$-statistic value; all errors refer to the $90 \%$ confidence level.

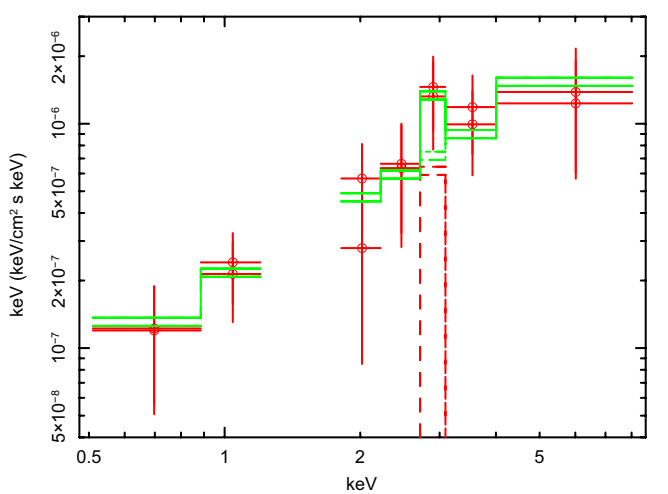

Fig. 4. The XMM-Newton MOS X-ray spectrum of CDFS-398, together with the best-fit power-law model. The binning is such that there are about 15 net counts per MOS1 or MOS2 bin. The source counts amount to $30 \%$ of the total (source+background) counts.

the degeneracies on the SED decomposition could play a crucial role, that is, in some cases the combination of different tori with star-forming models yield equally good fits, although the relative torus contribution changes. These degeneracies will be significantly overcome with the use of Herschel data, which will be able to provide tight constraints on the star-forming contribution. When we reject the sources with a low torus contribution, $<30 \%$, we are left with 14 low- $L_{X} / L_{6 \mu \mathrm{m}}$ sources (see Table 5). Out of these, six are Compton-thick according to the XMM-Newton spectroscopy (NGC 1068, NGC 1320, NGC 4968, NGC 6552, NGC 7479, NGC 7674).

\subsection{CDFS}

In most cases (seven out of nine), the torus component is the dominant contributor (over $80 \%$ ) to the $6 \mu \mathrm{m}$ luminosity. In the case of CDFS-382, the SED decomposition shows that the torus contribution is negligible and hence the $L_{\mathrm{X}} / L_{6 \mu \mathrm{m}}$ classification is problematic. Yet another source (CDFS-407) has a low (30\%) torus contribution to the $6 \mu \mathrm{m}$ emission. However, even taking into account the corrected $6 \mu \mathrm{m}$ luminosity originating from the torus, we find that this source would be marginally classified as a low $L_{X} / L_{6 \mu \mathrm{m}}$ ratio source.

In conclusion, there are eight low $L_{\mathrm{X}} / L_{6 \mu \mathrm{m}} \mathrm{AGN}$ in the CDFS, among the 283 luminous AGN $\left(L_{2-10 \mathrm{keV}}>10^{42} \mathrm{erg} \mathrm{s}^{-1}\right)$, after exclusion of CDFS-382 which has a zero torus contribution. At face value, that is assuming that all sources are
Table 5. Numbers of low $L_{\mathrm{X}} / L_{6 \mu \mathrm{m}}$ AGN (intrinsic $L_{\mathrm{X}}>10^{42} \mathrm{erg} \mathrm{s}^{-1}$ ) in the various samples, assuming the Fiore et al. (2009) relation for a $3 \%$ reflection efficiency.

\begin{tabular}{lcccccc}
\hline \hline & \multicolumn{3}{c}{ Total } & \multicolumn{3}{c}{ Torus $>30 \%$} \\
& all & CT & non-CT & all & CT & non-CT \\
\hline Local & 22 & 10 & 12 & 14 & 6 & 8 \\
CDFS & 9 & 4 & 5 & $7(8)^{\dagger}$ & 4 & $3(4)^{\dagger}$ \\
\hline
\end{tabular}

Notes. ${ }^{()}$Source CDFS-407 has a low (30\%) torus contribution in the $6 \mu \mathrm{m}$ flux, but it would still be considered low $L_{\mathrm{X}} / L_{6 \mu \mathrm{m}}$ taking into account this contribution.

Compton-thick, this would correspond to a Compton-thick fraction of $3 \%$. In agreement with the results in the local sample, not all the low $L_{\mathrm{X}} / L_{6 \mu \mathrm{m}} \mathrm{AGN}$ appear to be associated with Compton-thick sources. On the basis of their flat spectra (which is indicative of reflection-dominated spectra), as well as the presence of strong $\mathrm{Fe} \mathrm{K} \alpha$ lines, we estimate that at least two sources are Compton-thick (CDFS-309 and CDFS-398). One of these (CDFS-309 at a redshift of $z \approx 2.6$ ) was reported as a Compton-thick source by Feruglio et al. (2011), while the other one (CDFS-398 at a redshift of $z=1.222$ ) is reported here for the first time. Another two (CDFS-95, CDFS-197) are heavily obscured $\left(N_{\mathrm{H}} \sim 10^{24} \mathrm{~cm}^{-2}\right)$ hence consistent with being Comptonthick. For these sources only photometric redshifts are available, thus the column densities remain somewhat uncertain.

The fraction of Compton-thick AGN mentioned above (between two and four out of 283 sources) should be considered only as a lower limit. This is because there could be many more Compton-thick AGN at lower luminosities. There are 85 additional candidates with low $L_{\mathrm{X}} / L_{6 \mu \mathrm{m}}$ luminosity ratio that have an intrinsic X-ray luminosity $L_{\mathrm{X}}<10^{42} \mathrm{erg} \mathrm{s}^{-1}$ in the $2-10 \mathrm{keV}$ band. Many of these could be associated with normal galaxies (e.g. Georgakakis et al. 2003, 2007; Tzanavaris et al. 2006), as well as low-luminosity AGN with no obscuration. For example, Georgakakis et al. (2010) find that many low-luminosity AGN in the sample of Terashima et al. (2002) have low X-ray to midIR luminosity ratios. Nevertheless, there are five sources among them that display evidence of a flat spectrum (see Table 4). In principle, these could be associated with heavily obscured nuclei. Unfortunately, as they have faint fluxes, it is difficult to establish whether the hard spectra are caused by a moderate column density, or a genuinely flat spectrum, which would be the 
trademark of a reflection-dominated Compton-thick AGN. There is no evidence of an $\mathrm{Fe} \mathrm{K} \alpha$ line in these spectra.

\subsection{Comparison with previous results in the CDFS}

Interestingly, two bona fide Compton-thick sources do not have low $L_{\mathrm{X}} / L_{6 \mu \mathrm{m}}$ ratios. These are the sources CDFS-176 and CDFS-265 at a spectroscopic redshift of $z=1.536$ and $z=3.700$, respectively. These sources were reported as Compton-thick by Norman et al. (2002), Tozzi et al. (2006), and Georgantopoulos et al. (2007). Recently, Comastri et al. (2011) confirmed the presence of Compton-thick nuclei on the basis of both flat spectra and high- $E W \mathrm{Fe} \mathrm{K} \alpha$ lines. The most likely explanation of a high X-ray to $6 \mu \mathrm{m}$ luminosity ratio is the large intrinsic dispersion in this relation. This can be clearly seen in Fig. 1. It appears that the Compton-thick AGN in Comastri et al. (2011) have a relatively low $6 \mu \mathrm{m}$ luminosity compared to sources such as CDFS-309. Hence, only the last source lies below the Compton-thick line of Fig. 3. In any case, the existence of Compton-thick AGN with high $L_{X} / L_{6 \mu}$ m ratios certainly poses problems for the completeness of the $L_{X} / L_{6 \mu}$ method in identifying Compton-thick AGN.

In addition, Tozzi et al. (2006) identified a number of Compton-thick candidates on the basis of their flat spectra in the 1 Ms observations of the CDFS (see also Georgantopoulos et al. 2007). All these candidates, with the exception of CDFS-178, have high $L_{X} / L_{6 \mu \mathrm{m}}$ luminosity ratios (see Fig. 3). This source, at a spectroscopic redshift of $z=0.735$, is among our low X-ray luminosity sources that have a flat X-ray spectrum.

\section{Summary and conclusions}

We have assessed the efficiency of the low $L_{\mathrm{X}} / L_{6 \mu \mathrm{m}}$ ratio technique for identifying Compton-thick AGN. The principle behind this method is that in Compton-thick sources, the X-ray restframe luminosity, is diminished by almost two orders of magnitude while the $6 \mu \mathrm{m}$ luminosity remains unattenuated.

The advantages of our present approach compared to previous applications of the $L_{\mathrm{X}} / L_{6 \mu \mathrm{m}}$ method are the following:

- We have used a "calibration" sample in the local Universe. This consists of a sample for which high-quality XMMNewton observations are available, (Brightman \& Nandra $2011 \mathrm{~b})$ thus with a priori knowledge of the fraction of Compton-thick sources.

- At higher redshift, we have employed the most sensitive $\mathrm{X}$-ray observations available, namely the $4 \mathrm{Ms}$ Chandra observations, combined with the $3 \mathrm{Ms} X M M-N e w t o n$ observations in the CDFS (Comastri et al. 2011).

- We have derived the X-ray luminosities using X-ray spectral fits instead of the usually employed X-ray fluxes in combination with an average X-ray spectral index.

- We have used an SED decomposition to ascertain whether the nuclear contribution is not contaminated by significant amounts of star-forming emission.

Our conclusions can be summarised as follows:

- In the local sample, the vast majority (ten out of eleven) of the Compton-thick AGN appear to have low $L_{X} / L_{6 \mu \mathrm{m}}$ ratios. However, the converse is not true, i.e. a large number of low $L_{\mathrm{X}} / L_{6 \mu \mathrm{m}}$ sources are probably not Compton-thick. This implies that the efficiency of the $L_{\mathrm{X}} / L_{6 \mu \mathrm{m}}$ method for selecting Compton-thick sources is $50 \%$.
- In the CDFS, we have selected eight low $L_{X} / L_{6 \mu \mathrm{m}} \mathrm{AGN}$ with intrinsic luminosities $L_{\mathrm{X}}>10^{42} \mathrm{erg} \mathrm{s}^{-1}$ (after excluding one source where the $6 \mu \mathrm{m}$ emission comes from star-formation). One of these (CDFS-309 at a redshift of $z \approx 2.6$ ) had previously been shown to host a Compton-thick nucleus, whereas for another one (CDFS-398 at $z=1.222$ ) we have argued here for the first time that it is Compton-thick.

- We have been unable to confirm that a large fraction of the low $L_{\mathrm{X}} / L_{6 \mu \mathrm{m}}$ CDFS sources are Compton-thick on the basis of the X-ray spectroscopy, but they are nevertheless highly obscured, having column densities above $10^{23} \mathrm{~cm}^{-2}$. This suggests that the $L_{\mathrm{X}} / L_{6 \mu \mathrm{m}}$ ratio cannot be used on its own to reliably classify sources as Compton-thick.

- Finally, we have found that at least two bona fide Comptonthick sources from Comastri et al. (2011) which do not appear to have low $L_{X} / L_{6 \mu \mathrm{m}}$ ratios, casting further doubt on the reliability of this method for selecting Compton-thick sources.

Acknowledgements. I.G. and A.C. acknowledge the Marie Curie fellowship FP7-PEOPLE-IEF-2008 Prop. 235285. A.C., R.G. and C.V. acknowledge receipt of ASI grants I/023/05/00 and I/88/06. N.C. acknowledges financial support from the Della Riccia foundation. The Chandra data used were taken from the Chandra Data Archive at the Chandra X-ray Center.

\section{References}

Aird, J., Nandra, K., Laird, E. S., et al. 2010, MNRAS, 401, 2531

Ajello, M., Rau, A., Greiner, J., et al. 2008, ApJ, 673, 96

Akylas, A., \& Georgantopoulos, I. 2009, A\&A, 500, 999

Alexander, D. M., Bauer, F. E., Brandt, W. N., et al. 2003, AJ, 126, 539

Alexander, D. M., Chary, R. R., Pope, A., et al. 2008, ApJ, 687, 835

Alexander, D. M., Bauer, F. E., Brandt, W. N., et al. 2011, ApJ, 738, 44

Arnaud, K. A. 1996, Astronomical Data Analysis Software and Systems V, ed. G. Jacoby, \& J. Barnes, ASP Conf. Ser., 101, 17

Ballantyne, D. R., Draper, A. R., Madsen, K. K., Rigby, J. R., \& Treister, E. 2011, ApJ, 736, 56

Bongiorno, A., Mignoli, M., Zamorani, G., et al. 2010, A\&A, 510, 56

Braito, V., Della Ceca, R., Piconcelli, E., et al. 2004, A\&A, 420, 79

Brandt, W. N., \& Hasinger, G. 2005, ARA\&A, 43, 827

Brightman, M., \& Nandra, K. 2011a, MNRAS, 413, 1206

Brightman, M., \& Nandra, K. 2011b, MNRAS, 414, 384

Brunner, H., Cappelluti, N., Hasinger, G., et al. 2008, A\&A, 479, 283

Burlon, D., Ajello, M., Greiner, J., et al. 2011, ApJ, 728, 58

Catdamone, C. N., van Dokkum, P. G., Urry, C. M., et al. 2010, ApJS, 189, 270

Cash, W. 1979, ApJ, 228, 939

Chary, R., \& Elbaz, D. 2001, ApJ, 556, 562

Churazov, E., Sunyaev, R., Revnivtsev, M., et al. 2007, A\&A, 467, 529

Comastri, A. 2004, ASSL, 308, 245

Comastri, A., Iwasawa, K., Gilli, R., et al. 2010, ApJ, 717, 787

Comastri, A., Ranalli, P., Iwasawa, K., et al. 2011, A\&A, 526, L9

Daddi, E., Alexander, D. M., Dickinson, M., et al. 2007, ApJ, 670, 173

Damen, M., Labbé, I., van Dokkum, P. G., et al. 2011, ApJ, 727, 1

Dickey, J. M., \& Lockman, F. J. 1990, ARA\&A, 28, 215

Donley, J. L., Rieke, G. H., Alexander, D. M., Egami, E., \& Pérez-González, P. G. 2010, ApJ, 719, 1393

Eckart, M. E., McGreer, I. D., Stern, D., Harrison, F. A., \& Helfand, D. J. 2010, ApJ, 708, 584

Feruglio, C., Daddi, E., Fiore, F., et al. 2011, ApJ, 729, L4

Fiore, F., Grazian, A., Santini, P., et al. 2008, ApJ, 672, 94

Fiore, F., Puccetti, S., Brusa, M., et al. 2009, ApJ, 693, 447

Frontera, F., Orlandini, M., \& Landi, R. 2007, ApJ, 666, 86

Fukazawa, Y., Hiragi, K., Mizuno, M., et al. 2011, ApJ, 727, 19

Georgakakis, A., Hopkins, A. M., Sullivan, M., et al. 2003, MNRAS, 345, 939

Georgakakis, A., Rowan-Robinson, M., Babbedge, T. S. R., \& Georgantopoulos, I. 2007, MNRAS, 377, 203

Georgakakis, A., Rowan-Robinson, M., Nandra, K., et al. 2010, MNRAS, 406, 420

Georgantopoulos, I., \& Akylas, A. 2010, A\&A, 509, A38

Georgantopoulos, I., Georgakakis, A., \& Akylas, A. 2007, A\&A, 466, 823

Georgantopoulos, I., Georgakakis, A., Rowan-Robinson, M., \& Rovilos, E. 2008, A\&A, 484, 671 
Georgantopoulos, I., Akylas, A., Georgakakis, A., \& Rowan-Robinson, M. 2009, A\&A, 507, 747

Georgantopoulos, I., Rovilos, E., Xilouris, E. M., Comastri, A., \& Akylas, A. 2011, A\&A, 526, A86

Giacconi, R., Zirm, A., Wang, J., et al. 2002, ApJS, 139, 369

Gilli, R., Comastri, A., \& Hasinger, G. 2007, A\&A, 463, 79

Gilli, R., Vignali, C., Mignoli, M., et al. 2010, A\&A, 519, A92

Gilli, R., Su, J., Norman, C., et al. 2011, ApJ, 730, L28

Goulding, A. D., Alexander, D. M., Mullaney, J. R., et al. 2011, MNRAS, 411, 1231

Hao, L., Strauss, M. A., Fan, X., et al. 2005, AJ, 129, 1795

Iwasawa, K., Matt, G., Fabian, A. C., et al. 2001, MNRAS, 326, 119

La Franca, F., Fiore, F., Comastri, A., et al. 2005, A\&A, 635, 864

Luo, B., Bauer, F. E., Brandt, W. N., et al. 2008, ApJS, 179, 19

Luo, B., Brandt, W. N., Xue, Y. Q., et al. 2010, ApJS, 187, 560

Luo, B., Brandt, W. N., Xue, Y. Q., et al. 2011, ApJ, in press [arXiv: 1107.3148]

Lutz, D., Maiolino, R., Spoon, H. W. W., \& Moorwood, A. F. M. 2004, A\&A, 418,465

Magnelli, B., Elbaz, D., Chary, R. R., et al. 2009, A\&A, 496, 57

Maiolino, R., Shemmer, O., Imanishi, M., et al. 2007, A\&A, 468, 979

Marconi, A., Risaliti, G., Gilli, R., et al. 2004, MNRAS, 351, 169

Matt, G., Bianchi, S., Guainazzi, M., \& Molendi, S. 2004, A\&A, 414, 155

Merloni, A., \& Heinz, S. 2008, MNRAS, 388, 1011

Moran, E. C., Lehnert, M. D., \& Helfand, D. J. 1999, ApJ, 526, 649

Moretti, A., Pagani, C., Cusumano, G., et al. 2009, A\&A, 493, 501

Norman, C., Hasinger, G., Giacconi, R., et al. 2002, ApJ, 571, 218

Paltani, S., Walter, R., McHardy, I. M., et al. 2008, A\&A, 485, 707

Polletta, M., Tajer, M., Maraschi, L., et al. 2007, ApJ, 663, 81
Rafferty, D. A., Brandt, W. N., Alexnader, D. M., et al. 2011, ApJ, in press [arXiv: 1108.3229]

Richards, G. T., Lacy, M., Storrie-Lombardi, L. J., et al. 2006, ApJS, 166, 470 Rosenthal, D., Bertoldi, F., \& Drapatz, S. 2000, A\&A, 356, 705

Rush, B., Malkan, M. A., \& Spinoglio, L. 1993, ApJS, 89, 1

Sazonov, S., Krivonos, R., Revnivtsev, M., Churazov, E., \& Sunyaev, R. 2008 A\&A, 482, 517

Silva, L., Maiolino, R., \& Granato, G. L. 2004, MNRAS, 355, 973

Skrutskie, M. F., Cutri, R. M., Stiening, R., et al. 2006, AJ, 131, 1163

Soifer, B. T., Helou, G., \& Werner, M. 2008, ARA\&A, 46, 201

Soltan, A. 1982, MNRAS, 200, 115

Taylor, E. N., Franx, M., van Dokkum, P. G., et al. 2009, ApJS, 183, 295

Terashima, Y., Iyomoto, N., Ho, L. C., \& Ptak, A. F. 2002, ApJS, 139, 1

Tozzi, P., Gilli, R., Mainieri, V., et al. 2006, A\&A, 451, 457

Treister, E., Urry, C. M., \& Virani, S. 2009a, ApJ, 696, 110

Treister, E., Cardamone, C. N., Schawinski, K., et al. 2009b, ApJ, 706, 535

Tueller, J., Mushotzky, R. F., Barthelmy, S., et al. 2008, ApJ, 681, 113

Tzanavaris, P., Georgantopoulos, I., \& Georgakakis, A. 2006, A\&A, 454, 447

Ueda, Y., Akiyama, M., Ohta, K., \& Miyaji, T. 2003, ApJ, 598, 886

Ueda, Y., Eguchi, S., \& Terashima, Y. 2007, ApJ, 664, L79

Vignali, C., Alexander, D. M., \& Comastri, A. 2004, MNRAS, 354, 720

Winter, L. M., Mushotzky, R. F., Reynolds, C. S., \& Tueller, J. 2009, ApJ, 690, 1322

Wolf, C., Wisotzki, L., Borch, A., et al. 2003, A\&A, 408, 499

Wu, Y., Charmandaris, V., Huang, J., Spinoglio, L., \& Tommasin, S. 2009, ApJ, 701, 658

Xue, Y. Q., Luo, B., Brandt, W. N., et al. 2011, ApJS, 195, 10

Yaqoob, T. 1997, ApJ, 479, 184

Yaqoob, T., \& Murphy, K. 2011, MNRAS, 412, 835 